\title{
How to Avoid Making Your Systematic Review a Conventional Review Article
}

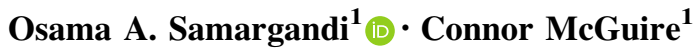

Received: 15 December 2020/ Accepted: 6 February 2021/Published online: 19 February 2021

(C) Springer Science+Business Media, LLC, part of Springer Nature and International Society of Aesthetic Plastic Surgery 2021

Level of Evidence $V$ This journal requires that authors assign a level of evidence to each article. For a full description of these Evidence-Based Medicine ratings, please refer to the Table of Contents or the online Instructions to Authors www.springer.com/00266.

Systematic reviews (SR) and their quantitative version, meta-analyses (MA), are an important retrospective method to efficiently and effectively utilize data from multiple original studies in the literature and synthesize studies to answer a specific clinical question $[1,2]$. This clinical question must be specified prior starting the search (a priori) in order to be able to systematically search and select relevant primary studies followed by extracting and analyzing data [2]. If designed and conducted correctly, SR and MA can meaningfully influence surgical clinical practice.

In recent years, there has been a rise in the popularity of SR and MA. Potential reasons include an increased likelihood to be read, highly citable, and more likely to be published [2]. Similar to other surgical disciplines, plastic surgery has witnessed an increase in the frequency of published SR and MA [2, 3]. However, a large proportion are not designed and/or performed properly [2, 3]. Improperly designed reviews have the ability to negatively influence patient care and surgical decision making. Understanding certain concepts and the inherent limitation of these studies is crucial to avoiding meaningless statistics and numerical summaries that can be intimidating to readers and reviewers alike.

\section{Osama A. Samargandi}

Osamargandi@dal.ca

1 Division of Plastic Surgery, Faculty of Medicine, Dalhousie University, Room 4447, Halifax Infirmary, 4th Floor, 1796 Summer Street, Halifax, NS B3H 3A7, Canada
Adding "systematic review" at the end of your title does not mean a SR was completed unless the truly necessary SR principles were followed. Knowing these principles will help differentiate the structure of SR from a conventional review article. The first key principle is to learn to generate a focused clinical question based on clinical practice. The most practical way to do this is by using the PICOS template, which stands for population, intervention, control, outcome, and study types [2-5]. To explain this further, we will use our previously published meta-analysis by McGuire and colleagues as an example [6]. Perhaps you wanted to evaluate the evidence for using Tranexamic Acid during aesthetic rhinoplasty before implementing this in your practice. First, define your population of interest and specify the age group (e.g., adult patients undergoing primary elective rhinoplasty). Second, decide what is your intervention and control/second intervention of interest (e.g., tranexamic acid vs normal saline). Third, define a clear primary outcome of interest (e.g., intraoperative blood loss and postoperative edema and ecchymosis). Finally, decide on the type of studies you are planning to include. The old epidemiological adage of "garbage in, garbage out" is important to consider when selecting what type of studies to include. Ideally, randomized controlled trials (RCTs) provide the bulk of included primary studies to provide the highest evidence-based conclusion. However, this type of study is not common in aesthetic plastic surgery; therefore, the best available controlled studies (case-controlled and cohort) are acceptable in our specialty [2-4].

Another principle that separates SR from conventional review articles is the necessity to plan a detailed, explicit, and replicable search strategy using pre-specified databases (e.g., MEDLINE, Embase, and Google Scholar) [2]. The search strategy should be transparent and completed details 
should be included in the manuscript. An important aspect of completing a systematic review/meta-analysis is involving a medical librarian to help build appropriate terms for searching the electronic and grey literature. As an example, here is a search query used for the study [6] that was completed examining the evidence for using tranexamic acid during rhinoplasty: "reconstruction" or "rhinoplasty" and "tranexamic acid" or "anti-fibrinolysis" and "bleeding" or "ecchymosis" or "bruising" or "edema" or "complications."

To help guide explicit reporting of your SR and MA, use the preferred reporting items for systematic reviews and meta-analyses (PRISMA checklist) and its flow diagram for a search strategy [7, 8]. The screening of potential included studies, study selection, and assessments should be done by at least two independent reviewers [2]. Any discrepancies can be resolved by a third independent reviewer or by consensus [2]. In addition to a detailed search strategy, a critical appraisal of final included articles should always be performed in a SR in order to assess study quality and the strengths of the conclusions [2]. Several methodological assessment tools are available, including the Cochrane Collaboration Risk of bias tool for RCTs [9], the MINORS criteria for non-randomized studies [10], and the AMSTAR2 instrument for SR/MA [11]. After the extraction of outcome data and quality assessments, data are typically synthesized either qualitatively with a SR, quantitatively with a MA, or by both methods. The decision to perform a MA requires an assessment of the heterogeneity (clinical, methodological, and statistical) of the extracted data [3]. Once deemed eligible, pooling of outcome can be possible. If completing a MA, authors should pay special attention to selecting a fixed or random effects modeling strategy as each has their pros and cons depending on the number of studies included in the analysis. For example, in McGuire and colleagues [6] a total of five high quality RCTs were included in the SR and qualitative data synthesis. However, four of these RCTs shared a homogenous outcome which was intraoperative blood loss and therefore a MA was properly performed for these four studies. Finally, the conclusion must be presented in parallel with the overall level of evidence and its quality $[2,3]$. These key methodological principles are summarized in Fig. 1.

It is noteworthy to mention that many of these steps are subjective to expert surgical discretion. For example, study inclusion can be very strict which will increase the internal validity and minimize heterogeneity while facilitating a MA [12]. Contrarily, broader criteria will likely include more studies and involve a wider patient distribution. This technique is commonly used in aesthetic plastic surgery as the volume of literature is small and typically heterogeneous $[3,4]$. Another step that requires epidemiological

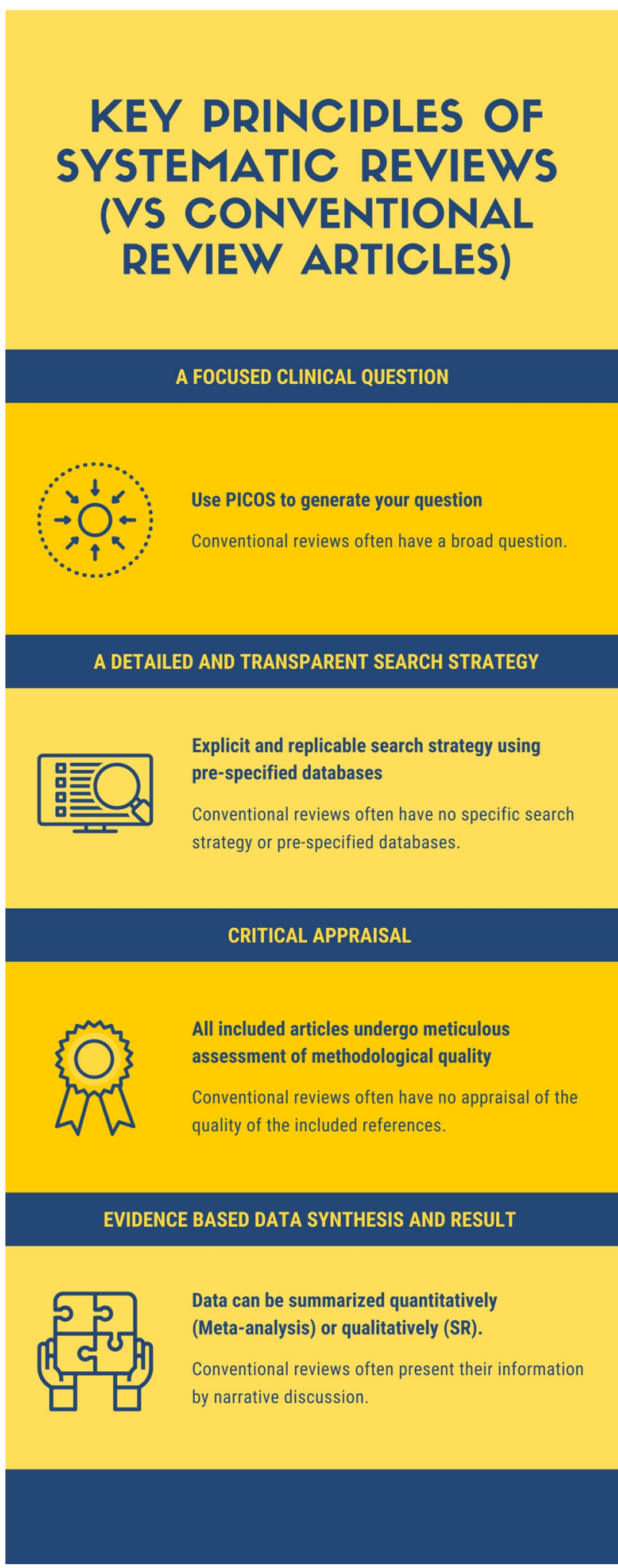

Fig. 1 Infographic summarizing key differences between systematic reviews and conventional reviews

judgment is the decision to combine outcomes from different studies. It is important to understand that any data 


\section{Tips to improve your next SR}

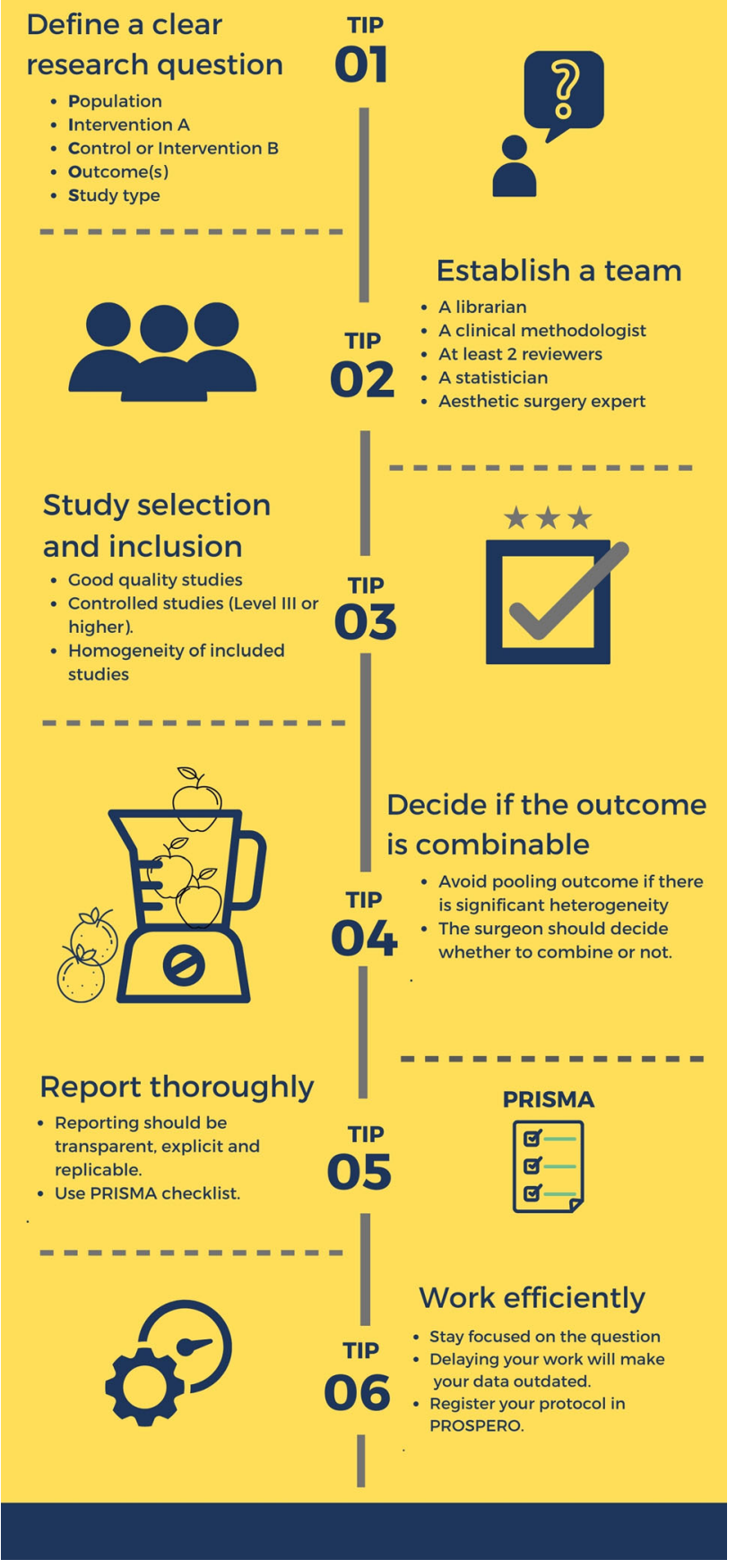

Fig. 2 Infographic summarizing key tips to perform systematic reviews properly. Systematic reviews (SR), meta-analyses (MA), preferred reporting items for systematic reviews and meta-analyses (PRISMA), International prospective register of systematic reviews (PROSPERO) https://www.crd.york.ac.uk/prospero/ from multiple studies can easily be pooled by a statistician and the results can be impressive for the naïve reader and/ or reviewers, regardless of their validity. The similarities between the population, interventions, and outcomes should be meticulously analyzed as this is arguably the most crucial decision when conducting a MA.

Future authors interested in conducting these studies, as well as reviewers, should understand the key differences between the conventional review article and SR/MA. We hope that this summary of key principles and tips (Figs. 1 and 2) will results in an enhanced quality SR and MA; hence, better evidence-based conclusions and patient outcomes.

\section{Compliance with Ethical Standards}

Conflict of interest The authors declare that they have no conflicts of interest to disclose.

Human Participants and Animals Rights This article does not contain any studies with human participants or animals performed by any of the authors.

Informed Consent For this type of study, informed consent is not required.

\section{References}

1. Bhandari M, Morrow F, Kulkarni AV, Tornetta P 3rd (2001) Meta-analyses in orthopaedic surgery. A systematic review of their methodologies. J Bone Joint Surg Am 83(1):15-24

2. Samargandi OA, Hasan H, Thoma A (2016) Methodological quality of systematic reviews published in the plastic and reconstructive surgery literature: a systematic review. Plast Reconstr Surg 137(1):225e-236e

3. McGuire C, Samargandi OA, Corkum J, Retrouvey H, Bezuhly M (2019) Meta-analyses in plastic surgery: can we trust their results? Plast Reconstr Surg 144(2):519-530

4. Coroneos CJ, Thoma A, Voineskos SH, Bain JR (2019) Discussion: Meta-analyses in plastic surgery: can we trust their results? Plast Reconstr Surg 144(2):531-532. https://doi.org/10.1097/ PRS.0000000000005881

5. Hulley SB, Cummings SR, Browner WS, Grady DG, Newman TB (2007) Designing clinical research, 3rd edn. Lippincott Williams and Wilkins, Philadelphia

6. McGuire C, Nurmsoo S, Samargandi OA, Bezuhly M (2019) Role of tranexamic acid in reducing intraoperative blood loss and postoperative edema and ecchymosis in primary elective rhinoplasty: a systematic review and meta-analysis. JAMA Facial Plast Surg 21(3):191-198. https://doi.org/10.1001/jamafacial.2018. 1737

7. Samargandi OA (2020) Improving reporting of research articles in aesthetic plastic surgery. Aesthetic Plast Surg 44(5):1935-1936. https://doi.org/10.1007/s00266-020-01884-y

8. Moher D, Liberati A, Tetzlaff J, Altman DG, PRISMA Group (2009) Preferred reporting items for systematic reviews and metaanalyses: the PRISMA statement. PLoS Med 6(7): e1000097

9. Higgins JP, Altman DG, Gøtzsche PC, Cochrane Bias Methods Group, Cochrane Statistical Methods Group (2011) The Cochrane Collaboration's tool for assessing risk of bias in randomised trials. BMJ 343:d5928 
10. Slim K, Nini E, Forestier D, Kwiatkowski F, Panis Y, Chipponi J (2003) Methodological index for non-randomized studies (minors): development and validation of a new instrument. ANZ J Surg 73(9):712-716. https://doi.org/10.1046/j.1445-2197.2003. 02748.x

11. Shea BJ, Reeves BC, Wells G et al (2017) AMSTAR 2: a critical appraisal tool for systematic reviews that include randomized or non-randomised studies of healthcare interventions, or both. BMJ 358:j4008
12. Thoma A, Eaves FF 3rd (2016) What is wrong with systematic reviews and meta-analyses: if you want the right answer, ask the right question! Aesthet Surg J 36(10):1198-1201. https://doi.org/ 10.1093/asj/sjw172

Publisher's Note Springer Nature remains neutral with regard to jurisdictional claims in published maps and institutional affiliations. 